\title{
Bottom-Up Elucidation of Glycosidic Bond Stereochemistry
}

Christopher J. Gray, ${ }^{\dagger}$ Baptiste Schindler, ${ }^{\ddagger}$ Lukasz G. Migas, ${ }^{\dagger}$ Martina Pičmanová, ${ }^{\S}$ Abdul R. Allouche, ${ }^{\ddagger}$ Anthony P. Green, ${ }^{\dagger}$ Santanu Mandal, ${ }^{\dagger}$ Mohammed S. Motawia, ${ }^{\S}$ Raquel Sánchez-Pérez, ${ }^{\S}$ Nanna Bjarnholt, ${ }^{\S}$ Birger L. Møller, ${ }^{\S}$ Anouk M. Rijs, ${ }^{\|}$Perdita E. Barran, ${ }^{*}, \dagger, \perp \odot$ Isabelle Compagnon, ${ }^{*},{ }^{\ddagger}, \#$ Claire E. Eyers, ${ }^{*} \times$ and Sabine L. Flitsch ${ }^{*}+\oplus$

${ }^{\dagger}$ School of Chemistry \& Manchester Institute of Biotechnology, The University of Manchester, 131 Princess Street, Manchester, M1 7DN, United Kingdom

${ }^{\ddagger}$ Institut Lumière Matière, UMR5306 Université Lyon 1-CNRS, Université de Lyon 69622 Villeurbanne Cedex, France

${ }^{\S}$ Plant Biochemistry Laboratory, Department of Plant and Environmental Sciences and Center for Synthetic Biology, University of Copenhagen, 40 Thorvaldsensvej, DK-1871 Frederiksberg C, Copenhagen, Denmark

"Radboud University, Institute for Molecules and Materials, FELIX Laboratory, Toernooiveld 7c, 6525ED Nijmegen, The Netherlands

${ }^{\perp}$ Michael Barber Centre for Collaborative Mass Spectrometry, Manchester Institute of Biotechnology, The University of Manchester, 131 Princess Street, Manchester, M1 7DN, United Kingdom

\#Institut Universitaire de France IUF, 103 Boulevard St. Michel, 75005 Paris, France

${ }^{\times}$Department of Biochemistry, Institute of Integrative Biology, University of Liverpool, Crown Street, Liverpool, L69 7ZB, United Kingdom

\section{Supporting Information}

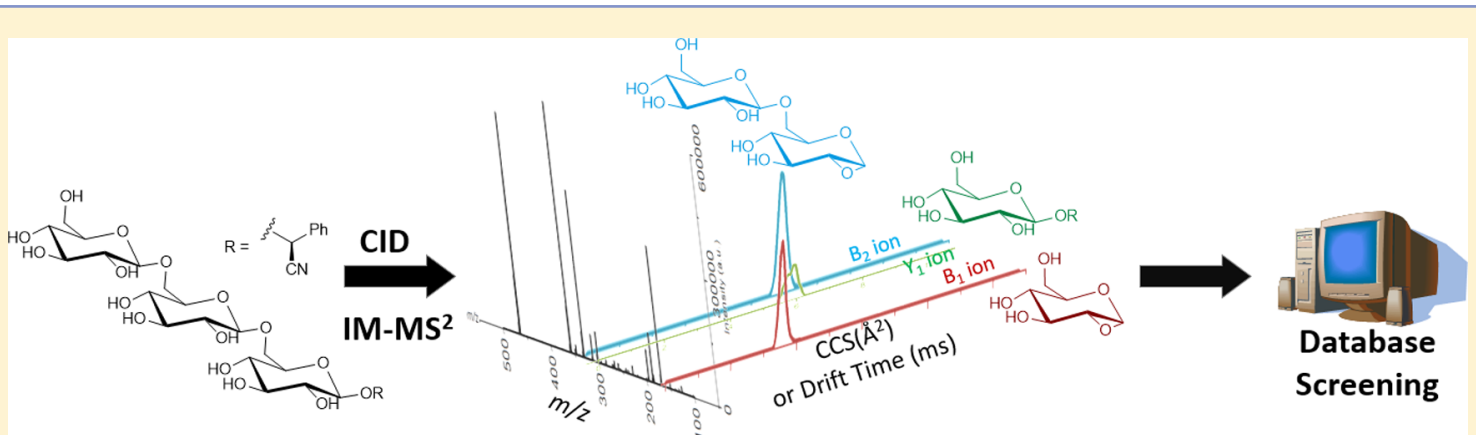

ABSTRACT: The lack of robust, high-throughput, and sensitive analytical strategies that can conclusively map the structure of glycans has significantly hampered progress in fundamental and applied aspects of glycoscience. Resolution of the anomeric $\alpha / \beta$ glycan linkage within oligosaccharides remains a particular challenge. Here, we show that "memory" of anomeric configuration is retained following gas-phase glycosidic bond fragmentation during tandem mass spectrometry $\left(\mathrm{MS}^{2}\right)$. These findings allow for integration of $\mathrm{MS}^{2}$ with ion mobility spectrometry (IM-MS ${ }^{2}$ ) and lead to a strategy to distinguish $\alpha$ - and $\beta$-linkages within natural underivatized carbohydrates. We have applied this fragment-based hyphenated MS technology to oligosaccharide standards and to de novo sequencing of purified plant metabolite glycoconjugates, showing that the anomeric signature is also observable in fragments derived from larger glycans. The discovery of the unexpected anomeric memory effect is further supported by IR-MS action spectroscopy and $a b$ initio calculations. Quantum mechanical calculations provide candidate geometries for the distinct anomeric fragment ions, in turn shedding light on gas-phase dissociation mechanisms of glycosidic linkages.

$\mathrm{C}$ arbohydrates possess unique structural complexity among the major classes of biomolecules (DNA/RNA/proteins), since glycosidic linkages between monomeric building blocks exist in different configurations, either as $\alpha$ - or $\beta$-anomers, which dictate structure and resultant biological function (Figure 1a). Current approaches to define the connectivity of glycans employing tandem mass spectrometry $\left(\mathrm{MS}^{2}\right)$ are intrinsically "blind" to such stereochemical information (Figure 1b). ${ }^{1-6}$ Resulting structural ambiguities can only be resolved by utilizing additional low-throughput strategies such as NMR or glycosidase digestion with chromatography, ${ }^{7}$ which typically offers significantly reduced sensitivity compared with MS-based methods. We and others have recently demonstrated that ion mobility-mass spectrometry (IM-MS) can be exploited for the separation of structural- and stereoisomers of glycopeptides ${ }^{1}$

Received: December 16, 2016

Accepted: March 28, 2017

Published: March 28, 2017 
a

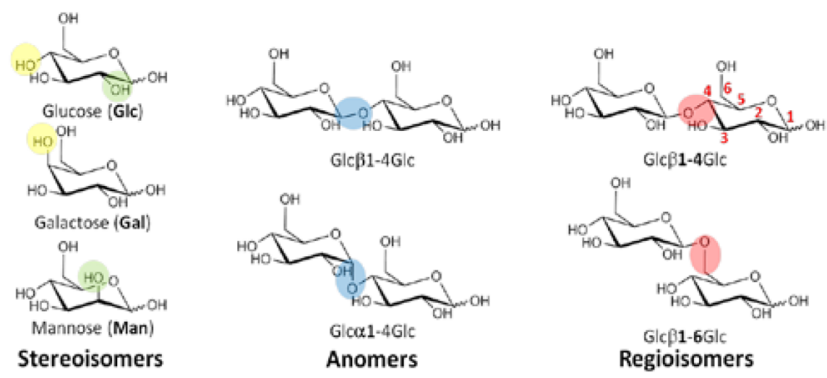

b- Tandem mass spectrometry

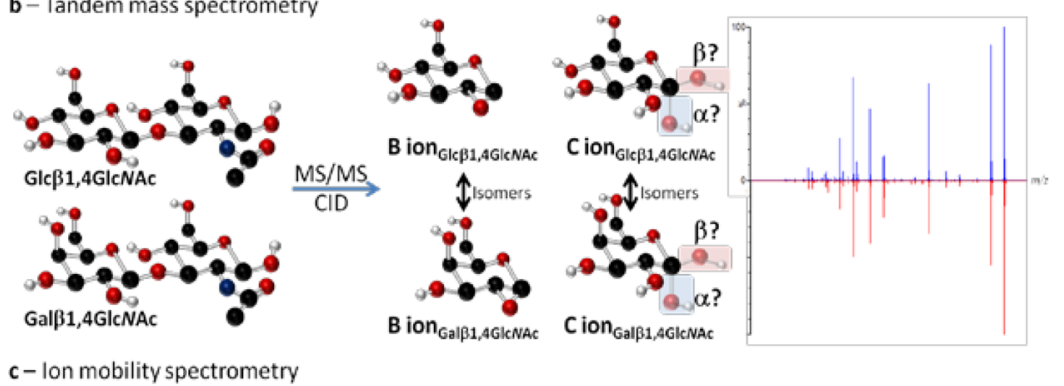

c-Ion mobility spectrometry
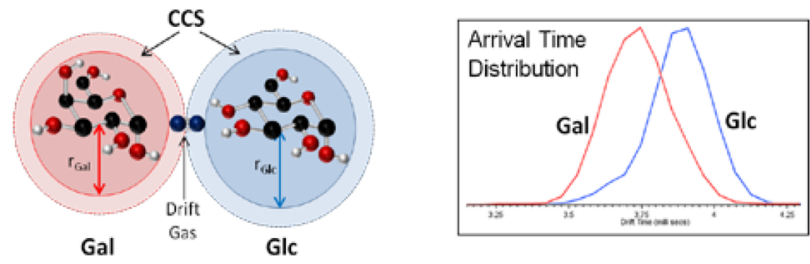

Gal
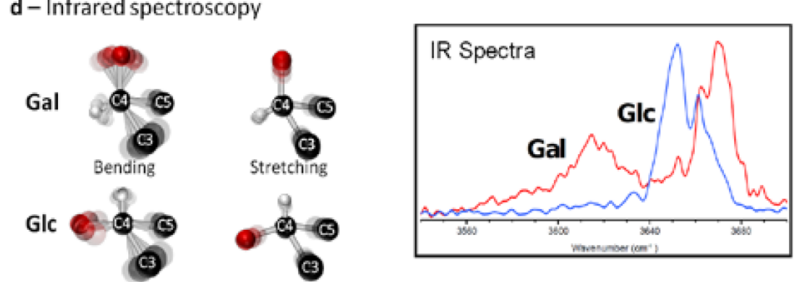

Figure 1. Carbohydrates possess a wealth of stereochemical information arising from the stereochemistry of the monosaccharide unit and the stereochemistry and regiochemistry of the glycosidic bond (a) and gas-phase techniques that can be combined with mass spectrometry to characterize them $(b-d)$. Conventional tandem mass spectrometry $(\mathrm{MS})$ is intrinsically blind to such information, and therefore spectra of isomers can be indistinguishable (b). Integration of ion mobility (IM) spectrometry with MS offers the ability to discern stereochemical information on the monosaccharide building blocks through derivation of rotationally averaged collision cross sections (CCS) of specific product ions (c). ${ }^{1}$ Infrared (IR) with MS differentiates species based on their vibrational modes, which are dependent on the atomistic configuration of the analyte providing complementary gas-phase structural information to IM-MS (d). Combined IM, IR, and tandem MS generate specific stereochemical information, which could lead to comprehensive carbohydrate sequencing techniques.

and free glycans. ${ }^{1-4,8-15}$ While MS determines the mass/charge $(\mathrm{m} / z)$ of an analyte ion, IM spectrometry discriminates ions based on their mobility which is inversely proportional to the rotationally averaged collision cross section area (CCS)-tocharge ratio, an intrinsic property of an ion's structure. ${ }^{16}$ Previously, we have identified the monosaccharide sequence of glycans by comparing the mobility of mono- and disaccharide product ions to simple reference standards using IM-MS ${ }^{2}$ (Figure 1c). ${ }^{1}$ However, the key stereochemical data on the configuration of anomeric linkage between the monosaccharide units is currently not provided. A truly universal $\mathrm{MS}^{2}$-based sequencing platform would necessitate that memory of precursor glycosidic configuration is retained following glycosidic fragmentation, which has not been demonstrated previously.

Here we report for the first time that glycosidic fragments generated by collision-induced dissociation (CID) can contain an "anomeric signature" derived from the precursor. Significantly, these glycosidic product ions can be separated and identified by IM-MS ${ }^{2}$. To provide further evidence for this "anomeric memory effect", IR action spectroscopy-MS ${ }^{2}$ (IR$\mathrm{MS}^{2}$ ) was employed to validate the IM-MS ${ }^{2}$ data (Figure 1d). More generally, combining data from both IM-MS ${ }^{2}$ and IR-MS ${ }^{2}$ with $a b$ initio calculations is demonstrated to be a powerful strategy to define structural features of carbohydrates.

\section{METHODS}

Synthesis, materials, IRMPD setup, computational methods, and additional spectra are described within the Supporting Information. Purified plant glycosides were prepared following the protocol of Pičmanová et al. ${ }^{17}$

Traveling Wave Ion Mobility-Mass Spectrometry (TWIMS-MS) Separation of Glycans. Glycans were diluted to $5 \mathrm{pmol} / \mu \mathrm{L}$ with $1: 1$ methanol-water prior to analysis. To 
a
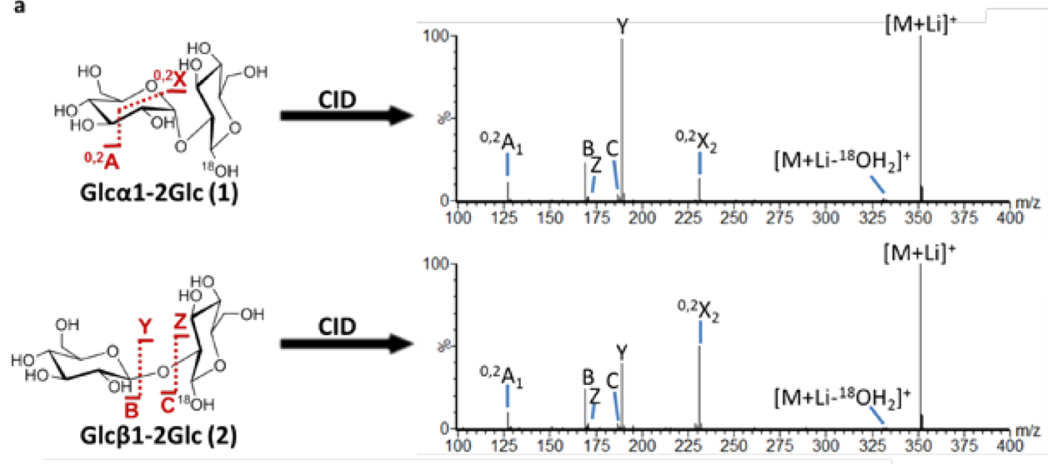

b

B Ion C Ion

Y lon
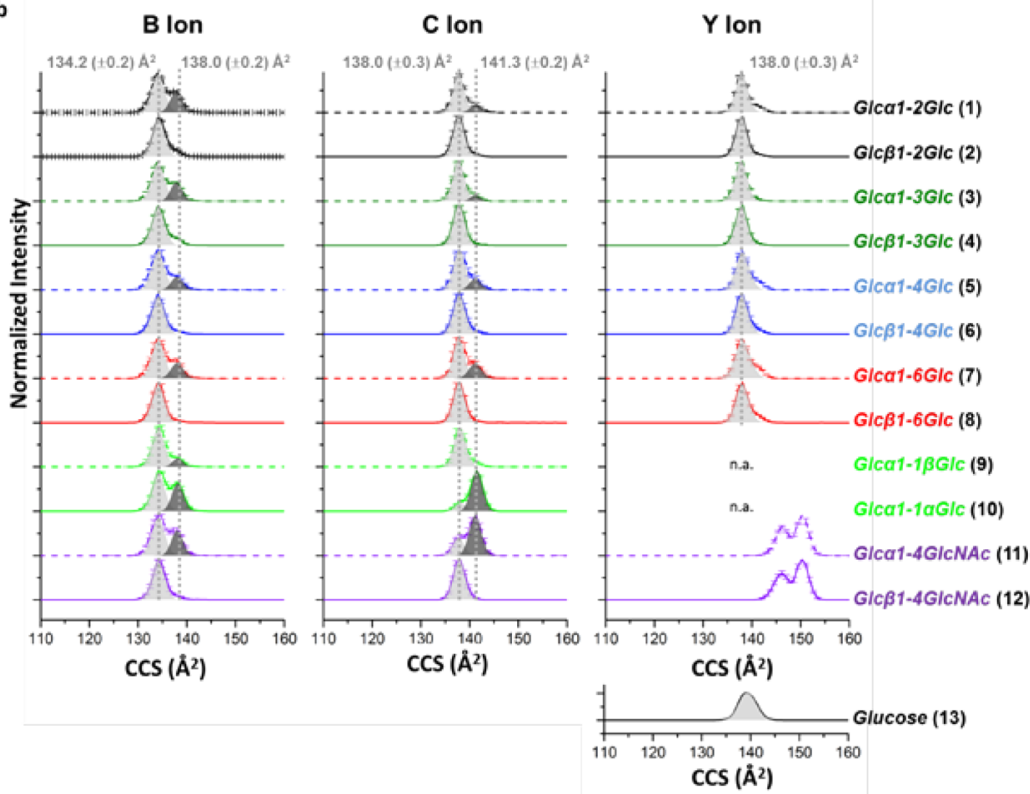

Figure 2. Illustration of the similarity between tandem mass spectra associated with disaccharide epimers Glc $\alpha 1-2 \mathrm{Glc} 1$ and Glc $\beta 1-2 \mathrm{Glc} 2$ compared to the ion-mobility (IM) arrival time distributions (ATDs) of selected glycosidic product ions. (a) Collision-induced dissociation (CID) of isomeric diglucosides generate similar tandem mass spectra especially for the $\alpha / \beta$-anomers. Disaccharides were selectively ${ }^{18} \mathrm{O}$-labeled at $\mathrm{C}_{1}$ of the reducing glycan by dissolution in ${ }^{18} \mathrm{O}$-water, allowing mass discrimination of the $\mathrm{C} / \mathrm{Y}$ - and $\mathrm{B} / \mathrm{Z}$-product ion pairs (Figure S1a,b). 4 was also treated with amyloglucosidase (Figure S2), which results in elimination of the shoulder from the B-ion ATD. Analytes were studied as lithiated ions which resulted in increased product ion yields compared with the corresponding sodiated precursors (Figure S3a). ${ }^{21-23} \mathrm{MS}^{2}$ spectra of 3-8 are shown in Figure S1c. (b) ATDs associated with B- and C-product ions generated by CID of the $\alpha$-glucose terminating precursor display more than one species compared to their $\beta$-glucose counterparts. It is important to note that Domon-Costello nomenclature ${ }^{20}$ for glycosidic product ions does not extend to nonreducing carbohydrates, hence B- and C-ions for 9 and 10 correspond to $\mathrm{m} / z 169$ and 187, respectively, despite it being highly likely the C-ion is formed by a Y-ion type mechanism. Displayed ATDs are the sum of three normalized and calibrated replicates. Intensity error bars are shown for all spectra whereas CCS error bars are only displayed for the B-ions of $\mathbf{1}$ and $\mathbf{2}$ for clarity, although the standard deviation is $<0.3 \AA^{2}$ for all shown ATDs. n.a.: not applicable.

study lithiated glycans, 10 equiv of lithium chloride was also doped into this solution. Samples were infused into a Synapt G2 HDMS (Waters, U.K.) by static nanoelectrospray ionization using pulled borosilicate emitters (World Precision Instruments; thin-wall capillary, 4 in. length, $1.2 \mathrm{~mm}$ o.d.). The capillary, cone voltage, and source temperature were typically set to $0.8-1.5 \mathrm{kV}, 40 \mathrm{~V}$, and $80{ }^{\circ} \mathrm{C}$, respectively. The IM traveling wave speed was set to $1200 \mathrm{~m} / \mathrm{s}$ and the wave height set at its maximum $40 \mathrm{~V}$. Nitrogen drift gas flow was set at 90 $\mathrm{mL} / \mathrm{min}$ for all experiments. The helium and argon flow was set to 180 and $2 \mathrm{~mL} / \mathrm{min}$, respectively, for the helium and trap cell. Collision-induced dissociation (CID) of quadrupole isolated precursor ions was induced in the trap region using an argon collision gas at a collision energy (CE) of $30 \mathrm{~V}$ unless specified otherwise. For energy-resolved IM-MS experiments, the trap collision energy was ramped at $5 \mathrm{~V}$ intervals (from $20 \mathrm{~V}$ until $80 \mathrm{~V}$ ) during the course of IM-MS acquisition.
Data were collected between $\mathrm{m} / z$ 50-1200 with a product ion tolerance of $\pm 50 \mathrm{ppm}$. The mass measurements were calibrated using product ions following CID of Glufibrinopeptide (trap CE, $35 \mathrm{~V}$ ) by infusion $(500 \mathrm{fmol} / \mu \mathrm{L}$; $1: 1$ acetonitrile-water, $0.1 \%(\mathrm{v} / \mathrm{v})$ formic acid) at a flow rate of $0.1 \mu \mathrm{L} / \mathrm{min}$. Drift times were calibrated to calculate ${ }^{\mathrm{TW}} \mathrm{CCS}_{\mathrm{N}_{2}}$ values of selected product ions by infusion of a stoichiometric mixture ( $1 \mathrm{pmol} / \mu \mathrm{L}^{-1}, 1: 1$ acetonitrile-water $0.1 \%$ formic acid) of small molecules (acetaminophen, verapamil, $\mathrm{N}$ ethylaniline, colchicine, and reserpine (all Sigma), whose CCS have been verified. ${ }^{18}$ Mass spectra and ATDs were recorded in triplicate and processed using MassLynx V4.1 (Waters, U.K.) and OriginPro 9.1 (OriginLabs), respectively. ATDs were calibrated and subsequently normalized to their maximum intensity. Gaussian distributions were fitted to these spectra, and the center of this fitted peak was taken as the peaks 
CCS. Reported errors are derived from the standard deviation of three calculated CCS replicates recorded on different days.

\section{RESULTS AND DISCUSSION}

Ion-Mobility Mass Spectrometry Analysis of Isomeric Diglucoside Standards. To assess whether the anomeric configuration is preserved in the product ion structures generated following collision-induced dissociation (CID), a series of reducing diglucoside standards (Glc $\alpha / \beta 1-2 / 3 / 4 / 6 \mathrm{Glc}$ ) $1-8$, which differ only in the regio- and stereochemistry of the glycosidic bond, were analyzed with a series of experiments that combined tandem mass spectrometry and ion mobility separation (Figure 2 and Figure S1). ${ }^{19}$ Samples were selectively ${ }^{18} \mathrm{O}$-labeled at $\mathrm{C} 1$ of the reducing glycan by dissolution in ${ }^{18} \mathrm{O}$ water to allow mass discrimination of the $\mathrm{C} / \mathrm{Y}$ - and $\mathrm{B} / \mathrm{Z}$ product ion pairs (Figure S1a,b). $\mathrm{MS}^{2}$ spectra associated with these diglucosides reveal that the anomers had different propensity to fragment at low collision energies. $\alpha$-Anomers were seen to dissociate more readily and the most abundant glycosidic product ions were $\mathrm{Y}$ - and $\mathrm{B}$-ions, with $\mathrm{C}$ - and $\mathrm{Z}$-ions being much less intense (Figure $2 \mathrm{a}$ and Figure S1c). Moreover, there is an apparent difference in the propensity of $\alpha$ or $\beta$ anomers to generate $\mathrm{B}$ - versus $\mathrm{Y}$-ions, with the $\mathrm{Y} / \mathrm{B}$ product ion ratio being significantly larger for $\alpha$-anomers. Interestingly and surprisingly, upon IM-MS analysis, two distinct arrival time distributions (ATDs) were observed for all B- and C-ions derived from $\alpha$-linked diglucosides, whereas a single ATD was predominant for the analogous $\beta$-linked structures independent of the precursor regiochemistry (Figure 2b). A minor contribution from a latter arriving species was observed for certain $\mathrm{B}$-ions derived from the $\beta$-anomers, although this never coincided with a counterpart for the C-ion (for example, 4). Upon treatment of this sample with amyloglucosidase, this shoulder for the $\beta$-anomers was eliminated suggesting contamination of the commercial samples with $\alpha$-linked glucosides (Figure S2). The higher fragmentation yield, the difference in B- versus Y-ion ratio, and the distinctive ATD immediately indicates that the anomers have different structures following IM-MS ${ }^{2}$ analysis. The observation that the B-ions produced from each anomer do not have identical ATDs is quite unexpected, since previously proposed B-ion structures $^{20,21}$ predict that the configuration at the $\mathrm{C} 1$ stereocenter is not retained during fragmentation. Collision cross sections, ${ }^{\mathrm{TW}} \mathrm{CCS}_{\mathrm{N}_{2}}$ (CCS recorded in $\mathrm{N}_{2}$ on a travelingwave IM-MS) derived from the ATDs (Figure 2b), allow us to compare the conformers from each glycosidic product ion. It is apparent that CCS values of $\beta$-linked diglucoside product ions (134.2 $\AA^{2}$ for B-ions, $138.0 \AA^{2}$ for C-ions) exhibit a high degree of similarity to the more intense conformer found in the IM$\mathrm{MS}^{2}$ analysis of $\alpha$-linked diglucosides (lower part of Figure 2b). Additionally, the later arriving, hence more extended species are notably less prevalent with increasing collision energy (Figure $\mathrm{S} 3 \mathrm{~b})$, suggesting that these larger structures are more labile.

ATDs of Y-ions derived from $\mathbf{1 - 8}$ are less diagnostic of the glycosidic linkage, suggesting that these product ion structures are solely dependent on the identity of reducing monosaccharide and not on the regio- or stereochemistry of the glycan bond (Figure 2b), as would be expected. Their ATDs are typically broader than their $\mathrm{C}$-ion equivalents and more typical of free-glucose 13 and many show a second larger conformer $(\mathbf{1}, 5$, and 6-8) irrespective of the precursor anomericity. Interestingly, the C-ion ATD derived from nonreducing Glc $\alpha 1-1 \beta$ Glc 9 strongly resembles C-ion ATDs from $\beta$-linked reducing diglucosides, suggesting that the $\alpha$ linkage is more labile. This is consistent with the increased product ion yields observed from $\alpha$-versus $\beta$-linked precursors. The ATD associated with the C-ions derived from Glc $\alpha 1$ $1 \alpha \mathrm{Glc} 10$ was dominated by the species with the larger CCS, an observation which may be explained by the increased lability of the glycosidic bond as a result of the neighboring acetal. Product ions from $\mathbf{9}$ and $\mathbf{1 0}$ were monitored as a function of the energy imparted (energy-resolved IM-MS ${ }^{2}$ ), ${ }^{13}$ revealing that the $\mathrm{C}$-ion with a larger ${ }^{\mathrm{TW}} \mathrm{CCS}_{\mathrm{N}_{2}}$ dissociates to yield a Band ${ }^{0,2} \mathrm{~A}_{1}$-ion and interestingly appears to be able to rearrange to the C-ion with a smaller ${ }^{{ }^{T W}} \mathrm{CCS}_{\mathrm{N}_{2}}$ (Figures S3b and S4). CID of all the reducing diglucosides $\mathbf{1 - 8}$ also generated ${ }^{0,2} \mathrm{~A}_{1}$ cross-ring fragments whose ${ }^{\mathrm{TW}} \mathrm{CCS}_{\mathrm{N}_{2}}$ are, as expected, identical, since they all contain identical nonreducing residues (Figure S5). Conveniently, the presence of other cross-ring fragments is dependent on the regiochemistry of the precursor (Table 1) providing additional information for sequence elucidation.

Table 1. ${ }^{\mathrm{TW}} \mathrm{CCS}_{\mathrm{N} 2}\left(\AA^{2}\right)$ of Studied Cross Ring Product Ions ${ }^{a}$

\begin{tabular}{llll} 
& \multicolumn{3}{c}{ cross ring fragments $\operatorname{CCS}_{\mathrm{N} 2}\left(\AA^{2}\right)$} \\
\cline { 2 - 4 } precursor ion & $m / z 229 / 231$ & $m / z 259 / 261$ & $m / z 289 / 291$ \\
Glc $\alpha 1-2 G l c$ 1 & $146.3( \pm 0.1)$ & & \\
& $153.8( \pm 0.0)$ & & \\
Glc $\beta 1-2 G l c$ 2 & $143.8( \pm 0.0)$ & & \\
& $148.3( \pm 0.1)$ & $150.7( \pm 0.7)$ & \\
Glc $\alpha 1$ 1-3Glc 3 & & $153.4( \pm 0.3)$ & \\
& & $151.1( \pm 0.1)$ & $158.0( \pm 0.1)$ \\
Glc $\beta 1-3 G l c$ 4 & & & $158.3( \pm 0.1)$ \\
Glc $\alpha 1-4 G l c 5$ & $146.4( \pm 0.1)$ & & $158.0( \pm 0.0)$ \\
Glc $\beta 1-4 G l c 6$ & $143.7( \pm 0.1)$ & $151.5( \pm 0.0)$ & $157.4( \pm 0.1)$ \\
Glc $\alpha 1-6 G l c 7$ & $143.7( \pm 0.0)$ & $150.7( \pm 0.0)$ & \\
Glc $\beta 1$-6Glc 8 & $142.6( \pm 0.0)$ &
\end{tabular}

${ }^{a}$ The standard deviation of the CCS obtained from each ATD following calibration to CCS with a set of standards run before and after each sample on each of three occasions is quoted in parentheses $(n=3)$.

Ion-Mobility-Mass Spectrometry Analysis of Anomeric Isomers. The resolution of anomers in the ATD of glycosidic product ions also extends to $\mathrm{N}$-acetylated disaccharides bearing a glucose (11 and 12), mannose (14 and 15), galactose (16-18), $N$-acetylglucosamine (19 and 20), or $\mathrm{N}$-acetylgalactosamine (21 and $\mathbf{2 2}$ ) residue at the nonreducing terminus (Figure S6). ATDs for B- and C-ions derived from $\mathbf{1 1}$ and $\mathbf{1 2}$ are consistent with those from 1-10 (Figure S6b). These trends were also observed under different ionization technique and after dissociation within the source region (Figure S7, discussed in greater detail within the Supporting Information). In contrast to the glucose containing disaccharides 1-12, C-ions derived from 14-18 and 21 and 22 give a single well-defined ATD that is dependent on both the identity of the monosaccharide residue and the stereochemistry of the glycosidic linkage, with the exception of 15 and 17 which cannot be distinguished by the C-ion ${ }^{\mathrm{TW}} \mathrm{CCS}_{\mathrm{N}_{2}}$ alone (Table 2 and Figure $\mathrm{S} 6 \mathrm{~b}-\mathrm{e})$. Anomers 19 and 20 also cannot be distinguished by their $\mathrm{C}$-ion ${ }^{\mathrm{TW}} \mathrm{CCS}_{\mathrm{N}_{2}}$ alone, but unlike 14-18 and 21 and 22 they consist of two well resolved species. B-ion ATDs from both 14 and 15 display two peaks whose ${ }^{\mathrm{TW}} \mathrm{CCS}_{\mathrm{N}_{2}}$ 
Table 2. ${ }^{\mathrm{TW}} \mathrm{CCS}_{\mathrm{N} 2}\left(\AA^{2}\right)$ of Glycosidic B- and C-Product Ions Including ${ }^{\mathrm{Tw}} \mathrm{CCS}_{\mathrm{N}_{2}}$ Data of Permethylated Precursors ${ }^{a}$

\begin{tabular}{|c|c|c|c|c|c|}
\hline \multirow[b]{2}{*}{ precursor ion } & \multirow[b]{2}{*}{ product ion(s) } & \multicolumn{2}{|c|}{ glycosidic fragments $\mathrm{CCS}_{\mathrm{N} 2}\left(\AA^{2}\right)$} & \multicolumn{2}{|c|}{ permethylated glycosidic fragments $\mathrm{CCS}_{\mathrm{N} 2}\left(\AA^{2}\right)$} \\
\hline & & B & $\mathrm{C}$ & B & $\mathrm{C}$ \\
\hline Glc $\alpha 1-4 \mathrm{GlcNAc} 11$ & $\alpha$-Glc & $\begin{array}{l}134.1( \pm 0.0) \\
137.9( \pm 0.2)\end{array}$ & $\begin{array}{l}137.9( \pm 0.2) \\
141.3( \pm 0.2)\end{array}$ & $\begin{array}{l}145.8( \pm 0.1) \\
153.4( \pm 0.3)\end{array}$ & $150.2( \pm 0.0)$ \\
\hline Glc $\beta 1-4 G l c N A c 12$ & $\beta$-Glc & $134.1( \pm 0.0)$ & $137.9( \pm 0.2)$ & $\begin{array}{l}145.5( \pm 0.0) \\
153.1( \pm 0.1)\end{array}$ & $149.4( \pm 0.1)$ \\
\hline Man $\alpha 1-4$ GlcNAc 14 & $\alpha$-Man & $\begin{array}{l}133.9( \pm 0.1) \\
137.9( \pm 0.1)\end{array}$ & $137.4( \pm 0.1)$ & $145.8( \pm 0.1)$ & $148.5( \pm 0.1)$ \\
\hline $\operatorname{Man} \beta 1-4$ GlcNAc 15 & $\beta$-Man & $\begin{array}{l}134.2( \pm 0.1) \\
138.0( \pm 0.3)\end{array}$ & $137.0( \pm 0.1)$ & $145.7( \pm 0.1)$ & $148.3( \pm 0.1)$ \\
\hline Galp $\alpha 1-4$ GlcNAc 16 & $\alpha-\mathrm{Gal}$ & $\begin{array}{l}134.1( \pm 0.1) \\
139.0( \pm 0.3)\end{array}$ & $138.2( \pm 0.1)$ & $145.1( \pm 0.1)$ & $148.9( \pm 0.1)$ \\
\hline Galp $\beta 1-4 G l c N A c 17$ & $\beta$-Gal & $\begin{array}{l}131.3( \pm 0.0) \\
134.2( \pm 0.1) \\
139.1( \pm 0.1)\end{array}$ & $137.0( \pm 0.1)$ & $144.9( \pm 0.1)$ & $148.5( \pm 0.1)$ \\
\hline Gal $f \beta 1-4 \mathrm{GlcNAc} 18$ & $\beta$-Galf & $\begin{array}{l}135.0( \pm 0.1) \\
138.6( \pm 0.0)\end{array}$ & $137.8( \pm 0.1)$ & $\begin{array}{l}145.3( \pm 0.7) \\
148.7( \pm 0.7)\end{array}$ & $149.1( \pm 0.0)$ \\
\hline GlcNAc $\alpha 1-4 \mathrm{Gal} 19$ & $\alpha$-GlcNAc & $\begin{array}{l}140.6( \pm 0.1) \\
143.8( \pm 0.1)\end{array}$ & $\begin{array}{l}146.0( \pm 0.1) \\
150.2( \pm 0.1)\end{array}$ & $\begin{array}{l}152.9( \pm 0.0) \\
156.3( \pm 0.2)\end{array}$ & - \\
\hline GlcNAc $\beta 1-6 \mathrm{Gal} 20$ & $\beta$-GlcNAc & $142.8( \pm 0.1)$ & $\begin{array}{l}146.1( \pm 0.2) \\
150.2( \pm 0.1)\end{array}$ & - & $157.8( \pm 0.4)$ \\
\hline GalNAc $\alpha 1-3 \mathrm{Gal} 21$ & $\alpha$-GalNAc & $\begin{array}{l}140.4( \pm 0.2) \\
143.4( \pm 0.1) \\
146.9( \pm 0.3)\end{array}$ & $145.7( \pm 0.1)$ & $\begin{array}{l}152.4( \pm 0.0) \\
155.0( \pm 0.1)\end{array}$ & - \\
\hline GalNAc $\beta 1-3 \mathrm{Gal} 22$ & $\beta$-GalNAc & $\begin{array}{l}140.3( \pm 0.2) \\
143.4( \pm 0.3) \\
147.0( \pm 0.0)\end{array}$ & $146.1( \pm 0.1)$ & $\begin{array}{l}152.6( \pm 0.0) \\
155.3( \pm 0.1)\end{array}$ & - \\
\hline $\operatorname{Ara} \alpha 1-5 \operatorname{Ara} 23$ & $\alpha$-Ara & $\begin{array}{l}128.9( \pm 0.1) \\
133.1( \pm 0.0)\end{array}$ & $131.9( \pm 0.1)$ & - & $139.7( \pm 0.1)$ \\
\hline Xyl $\beta 1-4 X y 124$ & $\beta$-Xyl & $\begin{array}{l}128.1( \pm 0.2) \\
133.2( \pm 0.1)\end{array}$ & $132.3( \pm 0.1)$ & $141.6( \pm 0.1)$ & $140.9( \pm 0.0)$ \\
\hline Fuc $\alpha 1-4$ GlcNAc 25 & $\alpha$-Fuc & $\begin{array}{l}131.0( \pm 0.2) \\
134.3( \pm 0.1)\end{array}$ & $\begin{array}{l}134.5( \pm 0.1) \\
139.6( \pm 0.0)\end{array}$ & $\begin{array}{l}141.8( \pm 0.0) \\
146.4( \pm 0.4)\end{array}$ & $143.3( \pm 0.0)$ \\
\hline Rha $\alpha 1-6$ Glc 26 & $\alpha$-Rha & $\begin{array}{l}131.8( \pm 0.1) \\
136.5( \pm 0.0)\end{array}$ & $\begin{array}{l}135.4( \pm 0.1) \\
140.5( \pm 0.1)\end{array}$ & $\times$ & $x$ \\
\hline
\end{tabular}

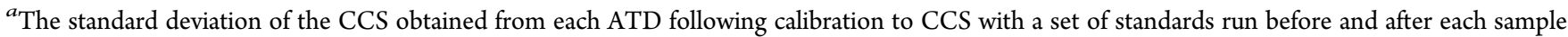
on each of three occasions is quoted in parentheses $(n=3)$. $p$ and $f$ correspond to the pyranose and furanose forms respectively; when not explicitly stated, the carbohydrate is in the pyranose form

are largely indistinguishable, while in contrast, B-ions derived from 17 exhibit an additional conformation $\left({ }^{\mathrm{TW}} \mathrm{CCS}_{\mathrm{N}_{2}} 131.3\right.$ $\left.\AA^{2}\right)$ to the two species in common with $16\left({ }^{\mathrm{TW}} \mathrm{CCS}_{\mathrm{N}_{2}} 134.0\right.$ and $139.0 \AA^{2}$ ). Three indistinguishable B-ion species were also observed for $\mathbf{2 1}$ and $\mathbf{2 2}$. However, the relative abundance of these three species is dependent on the anomeric configuration (Figure S6e) with the more extended conformer being significantly more abundant for 22. These data demonstrate that differences associated with ATDs of B- and/or C-ions can provide a convenient method to distinguish $\alpha$ - and $\beta$-anomers of hexoses and $N$-acetylhexosamines. Nonreducing terminal product ions of naturally occurring isomers 23 and 24 and 25 and $\mathbf{2 6}$ are also distinguishable.

Permethylation of $\mathbf{1 1}, \mathbf{1 2}$, and 14-26 reduces the observed differences in ATDs between anomeric pairs for B- and C-ions. For all B-ions, the distinction between anomeric pairs is lost (Table 2 RHS and Figure S6). For C ions small differences are still observed for selected examples such as 11 and 12. This suggests for at least the B-ions, the dissociation pathways that result in the formation of different anomeric dependent product ions species are blocked by permethylation. These data suggest that the hydroxyl groups are fundamentally important to the observed differences in product ions (perhaps through either nucleophilic or proton transfer mechanisms). However, when these hydroxyl groups are blocked, alternative dissociation pathways become favorable. We also observe that the difference between the epimeric nonreducing product ions is reduced for permethylated compared to the underivatized carbohydrates. This suggests the structural variability determined by IM arises from total conformational variability upon the stereochemistry of all the hydroxyl groups rather than its ability to measure the difference in stereochemistry at a single position. A possible explanation is that permethylation prevents such formation of these conformational families.

Infrared Multiple-Photon Dissociation of Gas-Phase Product Ion Isomers Structures. Following on from the initial unexpected discovery that anomeric information is retained after collisional dissociation, we were interested in using further independent spectroscopic techniques to characterize the fragments resulting from either $\alpha$ - or $\beta$ linkages. We thus investigated the use of infrared multiplephoton dissociation (IRMPD) spectroscopy coupled with MS. $^{24}$ Our current IRMPD setup does not allow selective 


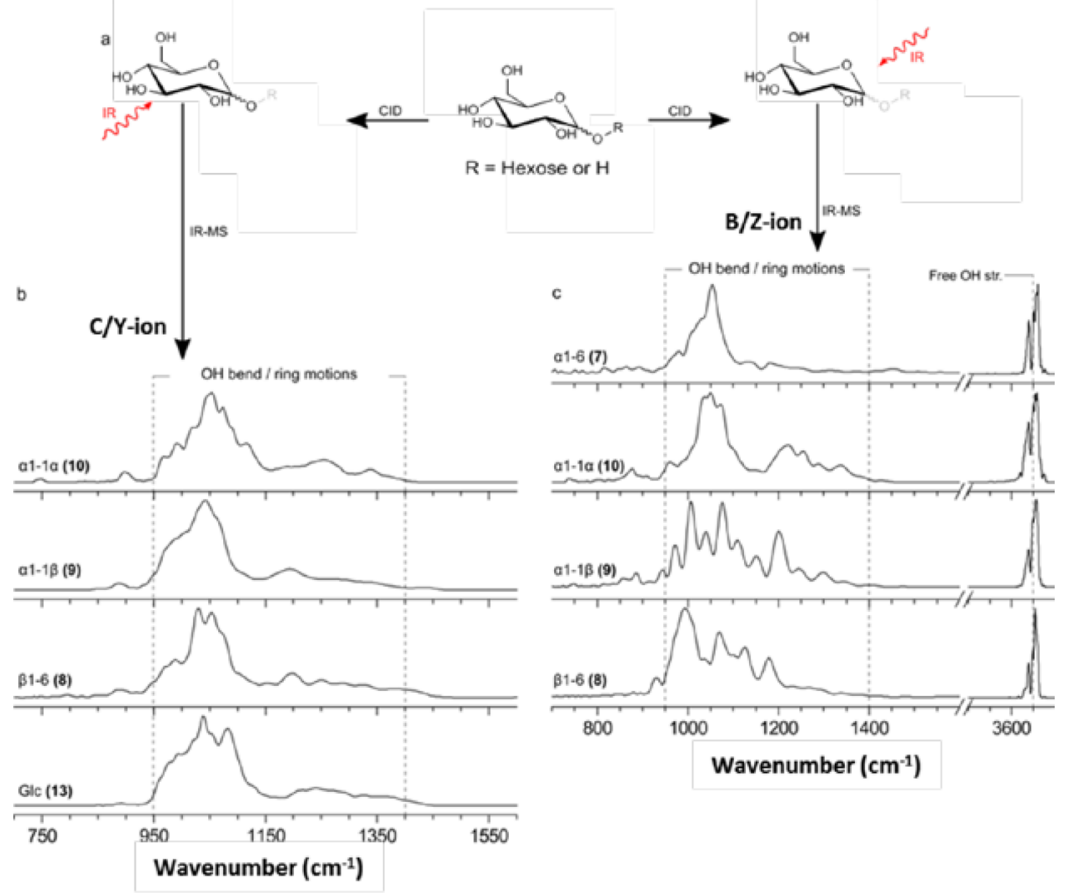

Figure 3. IRMPD spectra associated with B- and C/Y-glycosidic product ions. B- and C/Y-product ions at $m / z 169$ and 187 , respectively, were generated by collision-induced dissociation (CID) of precursors 7-10 and their vibrational spectra were recorded by IRMPD (a). Gas-phase IR action spectra of C-/Y-ions have an IR signature similar to glucose (13) (b). Specifically, the C-ion derived from $\alpha$-linked 10 displays an IR signature consistent with pure $\alpha$-glucopyranose, whereas others are primarily $\beta$-glucopyranose (Figure $4 \mathrm{~b}$ ). In contrast, each B-ion shows a distinctive IR signature (c), revealing that structural information is retained upon CID and supporting the observation of anomer-specific ATD in IM-MS. Features associated with atomic motions (free $\mathrm{OH}$ stretches, coordinated $\mathrm{OH}$ stretches, $\mathrm{OH}$ bends, and ring motions) are indicated.

spectroscopic analysis on conformationally selected ions. However, we were particularly interested to assess whether distinctive structural information can be retained upon CID, as suggested by the IM-MS experiments. The IRMPD spectra of the $\mathrm{C} / \mathrm{Y}$ - and $\mathrm{B}$-fragments of a subset of diglucosides (7-10) were recorded in both the near- and mid-IR ranges.

Following CID of 7-10 (unlabeled) and intact glucose 13 (Figure 3), IRMPD was performed on product ions at $m / z 169$ and 187.9 and 10 were specifically chosen as they are the only set of glucoside anomers whose $\mathrm{C}$-ion fragments exist primarily as either the smaller species or the extended conformer, respectively, thus circumventing issues associated with measuring chimeric action IRMPD spectra derived from these isomeric species. Unfortunately, no set of glucosides produces purely the extended species for the B-ion, therefore the measured action IR spectra of these species are likely chimeric with that of the shorter conformers.

$\mathrm{C} / \mathrm{Y}$-products ions (Figure $3 \mathrm{~b}$ ) share major structural features with glucose, in particular an intense, unresolved band centered around $1050 \mathrm{~cm}^{-1}$ and a low absorption region from 1150 to $1450 \mathrm{~cm}^{-1}$. The $\mathrm{C}$-ion derived from the purely $\alpha$ linked 10, however, displays two weak but distinctive features indicative of $\alpha$ character at 750 and $900 \mathrm{~cm}^{-1}$. These features are attenuated in glucose and those fragments derived from 8 and 9 , which indicate the presence of the $\beta$-anomer. B-ions (Figure 3c) show more variation in their spectroscopic features. In particular, the IRMPD spectrum of B-ions formed upon dissociation of $\alpha$-linked diglucosides (7 and 10) resembles that of glucose with a main band around $1050 \mathrm{~cm}^{-1}$. One may speculate that the chemical structure of these B-ions is close to that of glucose, for instance by maintaining a six-membered ring. In contrast, B-ions formed from $\beta$-linkage (8) or both contributions (9) show more complex spectra with several resolved bands from 970 to $1200 \mathrm{~cm}^{-1}$. These data support the formation of linkage-dependent product ions.

Strategy to Assign Structures to the Anomeric Product lons by IM-MS and IR-MS Combined. The IMMS experiments described above show a clear distinction between fragments from different glycosidic linkages, thus defining the anomeric stereochemistry. In order to understand this distinction, we need to assign candidate structures to the product ions. A well-established strategy to do this for gasphase ions is (i) to perform electronic structure calculations to determine the lowest energy conformers, (ii) calculate their CCS, and then (iii) compare this with experimental values. The drawback of this approach is that there are many putative geometries that will "fit" to a given measured CCS distribution. It can thus be difficult to differentiate between these multiple possibilities as not all of the calculated geometries will coincide with those obtained experimentally. To circumvent this, we used our mass selected infrared spectra for each product ion as an orthogonal experimental diagnostic. To obtain structural information, variable-wavelength infrared multiple-photon dissociation (IRMPD)-MS (IR-MS) is also commonly combined with high level $a b$ initio calculations. ${ }^{24}$ It is then possible to generate putative, calculated IR spectra from selected low energy structures for comparison with the experimental IR spectra. This approach has shown promising results for the discrimination of carbohydrate precursor ${ }^{25-30}$ and product ion diastereoisomers. ${ }^{31}$ Here we combine these two experimental strategies each providing unique empirical information to filter candidate structures (Figure 4a).

Starting from three putative geometries for $\mathrm{C}$-ions and 8 for B-ions, over 2000 conformations, respectively, were produced 


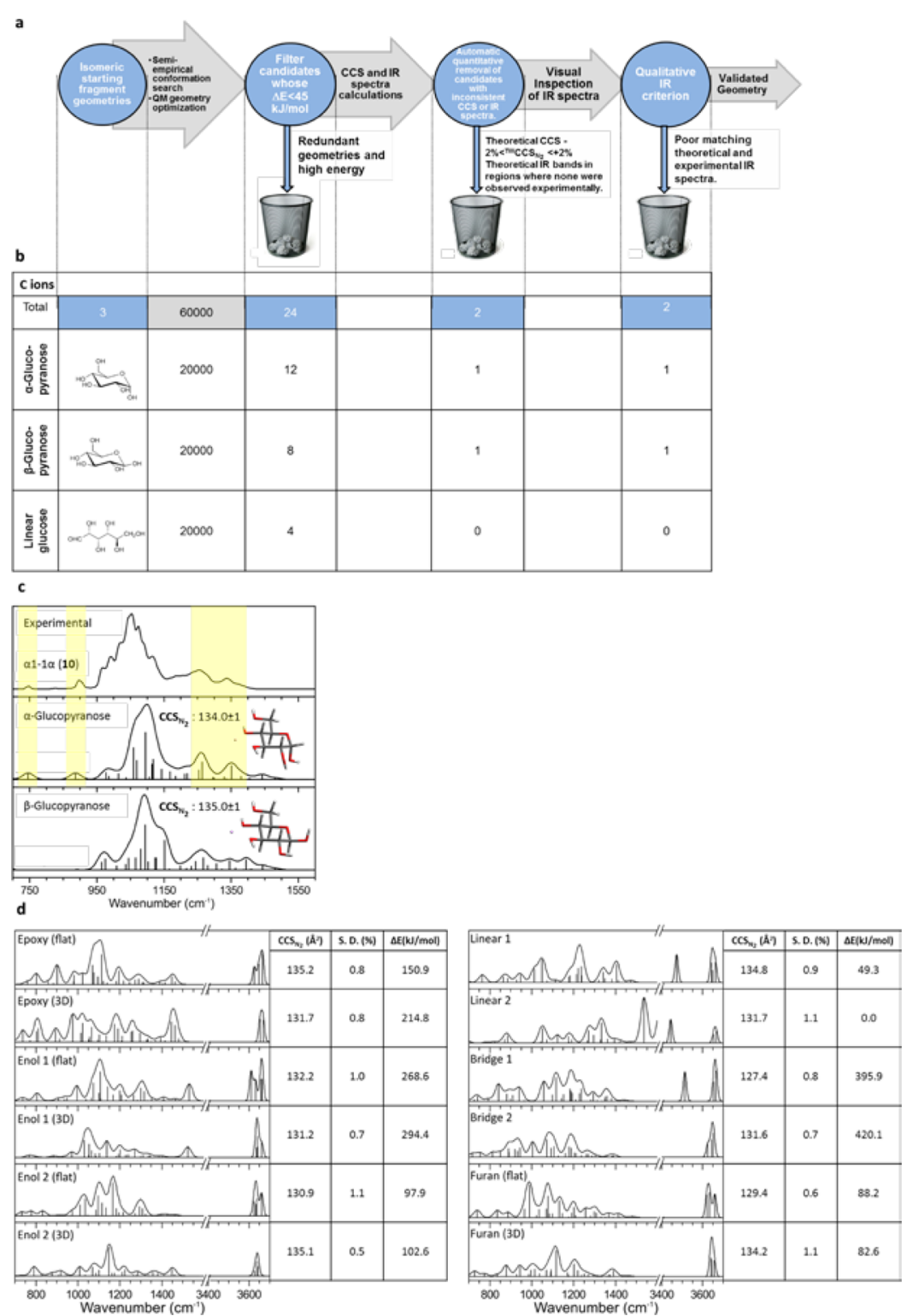

Figure 4. Combination of IM/IR-MS ${ }^{2}$ experimental and ab initio calculation strategies for generating potential gas-phase structures (a). Also shown are initial chemical structures for selected candidate of C-ions with examples of IR and CC data of low energy conformers (b, c). The number of potential gas-phase structures that meet the strategies criteria after each stage of selection is given (b). (c) Experimental spectra for the $\mathrm{C}$ ion for $\mathbf{1 0}$ and for comparison are the calculated spectra of $\alpha$ (middle) and $\beta$ (bottom) glucopyranose. Theoretical frequencies (bars) and convoluted spectra (line) calculated in the harmonic approximation at the DFT CAM-B3LYP/6-311G(2df,2pd) level of theory with scaling factors 0.964/0.943 for low frequency/high frequency are shown. The lowest energy structure is a ${ }^{4} \mathrm{C}_{1}$ conformation. Its anomeric configuration can be further identified via the two $\alpha$-specific features highlighted around 750 and $900 \mathrm{~cm}^{-1}$. Other energetic minima include ${ }^{1} \mathrm{C}_{4}$, boat and ring open due to the stabilizing effect of the lithium cation (not shown). Overview of the quantitative 2-criteria analysis of the B ions candidate structures (d). Candidate energetic minima produced by conformational exploration from 8 putative geometries: Epoxy, Enol 1, Enol 2, Linear 1, Linear 2, Bridge 1 Bridge 2, Furan; from which 2 additional families emerged from 4 of the starting structures here classified as "flat" or "3D". Two orthogonal quantitative criteria were applied (CCS, $130.0 \AA^{2}<$ Theoretical CCS < $142.0 \AA^{2}$; IR, absence of coordinated OH stretches) to eliminate irrelevant candidates. S.D., standard deviation (\%); $\Delta E$, relative energy $(\mathrm{kJ} / \mathrm{mol})$.

by semiempirical exploration of the conformational space at the PM7 level of theory. Nonredundant conformers were then optimized by quantum mechanics and sorted by energy. Theoretical spectra and theoretical CCS values were generated for structures with $\Delta E<45 \mathrm{~kJ} \mathrm{~mol}^{-1}$ and assessed against the experimental IRMPD spectra and measured CCS. The match between calculated CCS values and those found experimentally was used as the first pass filter to reject structures that did not fall within $2 \%$ of the experimentally determined values. Subsequently, a visual match between the calculated IR spectra from the remaining candidate geometries to that found experimentally was made. For the assessment of IR spectra of $\mathrm{C}$-ions, this visual inspection was sufficient given the modest number of low energy structures (Figure $4 \mathrm{c}$ and Figure S7). For the eight starting geometries for B-ions, a larger number of low energy conformations were produced (Figure S8). Two quantitative criteria for the assessment of their IR spectra were thus used: structures displaying optical activity between 3400 and $3550 \mathrm{~cm}^{-1}$ and/or intense features between 1400 and $1600 \mathrm{~cm}^{-1}$ were discarded, given that features were not 
observed experimentally in these regions. The representative calculated IR spectra and the corresponding CCS values are shown in Figure $4 \mathrm{~b}$ for visual matching to experimental data (Figures 2, 3c and Figure S9).

Combining IRMPD/IR-MS with IM-MS Data to Resolve Product Ion Structures. After exploration of the conformational space of lithiated glucose (Glc, 13), the IR spectra shown in Figure $3 \mathrm{~b}$ can be attributed to a glucopyranose ${ }^{4} \mathrm{C}_{1}$ form with $\mathrm{Li}^{+}$intercalated between $\mathrm{O} 3$ and $\mathrm{O} 4$. This is in contrast to their anionic equivalents that have been previously reported to exist in the open-chain hydroxyl-carbonyl form. ${ }^{27}$ Other energetic minima (for example, ${ }^{1} \mathrm{C}_{4}$, boat, linear) were discarded based on qualitative spectral matching.

The IR spectrum of the Y-ion, shown in the left panel of Figure $3 \mathrm{~b}$, of $\mathbf{8}$, strongly correlates with the $\beta$-glucopyranose structure (Figure $4 \mathrm{c}$ ) highlighting the preferential adoption of the $\beta$-glucopyranose structure in the gas-phase by reducing glycans. This is in agreement with observed equilibrium positions in solution-phase. Significantly, the nonreducing disaccharide 10 derived $\mathrm{C}$-ion displays two distinctive bands at 750 and $900 \mathrm{~cm}^{-1}$ which strongly match features within the theoretical spectrum for the $\alpha$-glucopyranose shown in Figure $3 \mathrm{~b}$. In contrast, $\mathrm{C}$-ions derived from 9 have features in common with the $\beta$-glucopyranose structures, suggesting that the $\alpha$ glycosidic bond preferentially dissociates as might be expected. The theoretical ${ }^{\mathrm{TW}} \mathrm{CCS}_{\mathrm{N}_{2}}$ of these two structures (135.0 and $137.0 \AA^{2}$ for $\beta$ - and $\alpha$-glucopyranose, respectively) fall within the recorded ${ }^{\mathrm{TW}} \mathrm{CCS}_{\mathrm{N}_{2}}$ distribution from experimental IM-MS data of ions derived from 9 and $\mathbf{1 0}$ (Figure 2). The combined IRMPD and IM-MS data provide strong evidence that the two resolved C-ion species derived from structures with nonreducing glucose residues are the $\beta$ - and $\alpha$-glucopyranose structures. Interconversion of $\alpha$ - and $\beta$-anomers during CID (and mobility separation) is therefore not prevalent for C-ions derived from lithiated $\mathbf{1 0}$ or the HexNAc derivative 11 which dissociate primarily to the $\alpha$-glucopyranose structure. However, at elevated collision energies, IM-MS reveals that this $\alpha$ glucopyranose structure can rearrange to the $\beta$-glucopyranose form. Although this conversion appears more prevalent for Cions derived from diglucosides $\mathbf{1}, \mathbf{3}, \mathbf{5}$, and 7 , the distributions of these structures are dependent upon the nature of the precursor glycosidic linkage, allowing this key stereochemical data to be determined. Careful control (and potential further optimization) of the CID and mobility separation conditions will thus minimize the effect of undesired conversion and increase reliable characterization of anomeric configuration. Finally, since the ATDs of C-ions derived from $\alpha$ - and $\beta$ mannosides/galactosides $\mathbf{1 4 - 1 8}$ and $\mathrm{N}$-acetylgalactosaminisides 21 and 22 consist of a single species with a drift time dependent on the anomeric linkage, it is appears that the barrier to gas-phase anomeric inversion is larger for these structures.

The B-ions derived from 7-10 all display distinctive IR spectra (Figure 3c). IR spectra of 7 and $\mathbf{1 0}$ appear more congested than $\mathbf{8}$ and $\mathbf{9}$, which is likely the result of coexisting isomeric structures as shown by their IM-MS ATDs. Structural assignment of $\mathrm{B}$-ions is thus more challenging due to this multiplicity of isomeric structures (see Figures S8 and S9). To extract structural information from the IR spectra shown in Figure $3 c$, we have quantitatively assessed energetic minima from each of eight candidate isomers (Figure S8) using a procedure based on two orthogonal structural criteria: matching experimental ion mobility data and diagnostic IR activity. In total, $76 \%$ of the candidate structures were discarded on the basis that their theoretical CCS values were incompatible with measured values and on mismatching $\mathrm{OH}$ stretching frequencies. All remaining candidates from each putative geometry were assessed by a traditional, qualitative IR pattern matching in the complementary spectral range $(\mathrm{OH}$ bending frequencies). Typical theoretical IR patterns for the candidate isomers are shown in Figure $4 \mathrm{~d}$ for comparison with the experimental IRMPD spectra Figure 3c. Among the remaining candidates, the best qualitative matches in the low energy range are found for $\mathbf{8}$ and $\mathbf{1 0}$ with furan and epoxy forms, respectively, implying that the fragmentation mechanism is precursor-dependent. It is evident from this approach that the distinction between some sugars (for example, $\alpha$-glucopyranose and $\beta$-glucopyranose) may be attributable to know deviations of computational methods in comparison to experiment, for this reason we advocate the combined use of IMS along with IR-MS (both supported by calculation) to best distinguish isomeric species.

Application to Plant Glycosides. In order to extend our studies from validated standards to novel biological glycoconjugates, the application of this hyphenated IM-MS ${ }^{2}$ method for de novo fragment based glycan sequencing was demonstrated using a selection of plant glycosides extracted from almond (Prunus dulcis (Mill.) D.A. Webb) and cassava (Manihot esculenta Crantz), whose structural elucidation has been extremely challenging. ${ }^{17} \mathrm{MS}^{2}$ analysis of reverse-phase C18 and phenylhexyl liquid chromatography (LC) separated fractions indicated the presence of a series of cyanogenic glycosides and their structural analogues but the limited amounts of sample did not allow for further structure elucidation, a problem frequently encountered with glycoside mixtures in biological materials

By comparing the ${ }^{\mathrm{TW}} \mathrm{CCS}_{\mathrm{N}_{2}}$ values of the B-, C-, and crossring fragments with synthetic standards (Figure S10), it was possible to assign the structure of one of these species to amygdalin (27). We further analyzed three distinct species of trisaccharide 28a, 28b, and 28c (Figure 5a) for which no synthetic standards were available. ${ }^{17}$ Terminal $\mathrm{B}$ - and C-ions derived from all three structures clearly indicated the presence of $\beta$-linked glucose units (Figure $5 \mathrm{~b}$ and Figure S11a-c). The cross-ring fragments ${ }^{\mathrm{TW}} \mathrm{CCS}_{\mathrm{N}_{2}}$ (or lack thereof) enabled unambiguous identification of the regiochemistry of the glycosidic linkages, with $\mathbf{2 8 a}, \mathbf{2 8 b}$, and $28 \mathrm{c}$ being the $\beta 1-6$, $\beta 1-4$, and $\beta 1-2$ isomers, respectively (Figure 5c). Finally, the ATDs of the $\mathrm{Y}_{2}$-ions reveal identical reducing termini to the precursor 27 (Figure S11d).

\section{CONCLUSION}

In conclusion, we have demonstrated that IM-MS ${ }^{2}$ can be used as a fragment-based method to characterize both primary glycan sequence and crucially the stereochemistry of the glycan linkages, since "memory" of anomeric configuration is retained following CID of lithiated precursors. Strong correlation of IMMS and IR-MS data for C-ions enables unambiguous identification of the gas-phase product ions to be ring-closed $\alpha$ - or $\beta$-glucopyranose. We also find that such a strong correlation cannot be readily elucidated for $\mathrm{B}$-ions given the presence of $\mathrm{m} / \mathrm{z}$ irresolvable isomeric species during the IR$\mathrm{MS}^{2}$ analysis, as confirmed by IM-MS ${ }^{2}$. We seek to further define these structures in future in-line IM-IR-MS ${ }^{2}$ experiments. Given the distinct fingerprints provided by IM-MS ${ }^{2}$, combined 
a
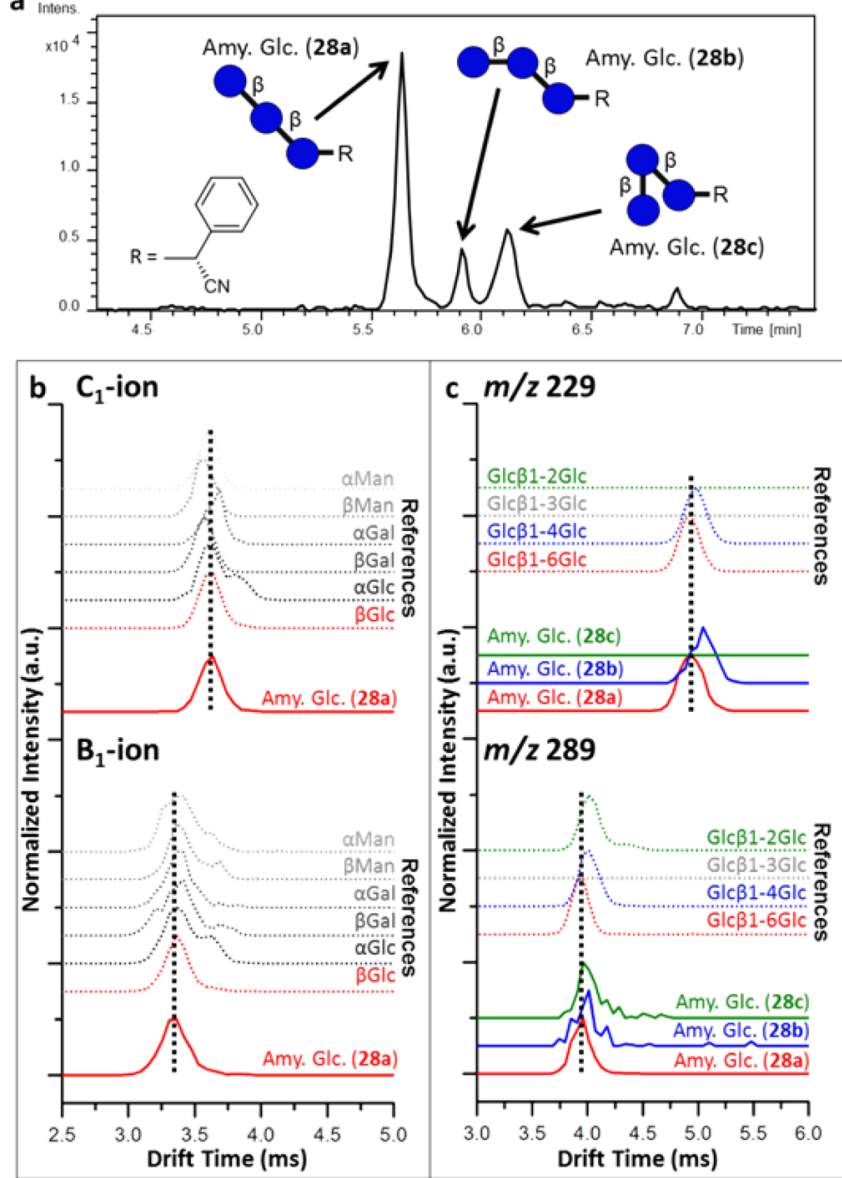

Figure 5. Extracted ion chromatogram (XIC) and selected arrival time distributions (ATDs) associated with amygdalin glucosides 28a, 28b, and 28c (2(R)-[ $\beta$-D-Glucopyranosyl-(1-x)- $\beta$-D-Glucopyranosyl-(1-6)$\beta$-D-glucopyranosyloxy]phenylacetonitrile) compared to standards ( $x$ is 2, 4, or 6). XIC associated with $m / z 642.0$ (amygdalin glucoside + $\mathrm{Na}^{+}$) reveal the presence of at least three isomers (a). The proposed $\mathrm{B}_{1}$ and $\mathrm{C}_{1}$ glycosidic product ions associated with amygdalin glucosides (28b and $28 \mathrm{c}$ omitted for clarity) clearly match that of $\beta$ Glc (b). Proposed cross-ring fragments ${ }^{0,4} \mathrm{~A}_{2}$ and ${ }^{0,2} \mathrm{~A}_{2}$ generated by collision-induced dissociation (CID) of $28 \mathrm{a}, \mathbf{2 8 b}$, and $28 \mathrm{c}$ clearly match those of Glc $\beta 1-6 \mathrm{Glc}$, Glc $\beta 1-4 \mathrm{Glc}$, and Glc $\beta 1-2 \mathrm{Glc}$, respectively (c). Glycoside structures within panel a are displayed in the consortium of Glycomics (CFG) format. The vertical black dashed line represents the peak center of the ATD associated with amygdalin glucoside 28a.

with the sensitivity and speed of the instrumentation, we envisage that the techniques reported here have the potential to lay the foundation for the development of generic carbohydrate sequencing platforms.

\section{ASSOCIATED CONTENT}

\section{S Supporting Information}

The Supporting Information is available free of charge on the ACS Publications website at DOI: 10.1021/acs.analchem.6b04998.

Additional experimental details and discussion, mass spectra, arrival time distributions, normalized intensities plots, scheme depicting product ion structures and their respective arrival time distributions, overlaid arrival time distributions, scheme depicting the initial chemical structures of the B-ion candidates and the number of potential conformers within each family, example results from the candidate computational structures compared with experiment, and reaction schemes (PDF)

\section{AUTHOR INFORMATION}

\section{Corresponding Authors}

*E-mail: perdita.barran@manchester.ac.uk.

*E-mail: isabelle.compagnon@univ-lyon1.fr.

*E-mail: claire.eyers@liverpool.ac.uk.

*E-mail: sabine.flitsch@manchester.ac.uk.

ORCID *

Baptiste Schindler: 0000-0002-7376-4154

Perdita E. Barran: 0000-0002-7720-586X

Isabelle Compagnon: 0000-0003-2994-3961

Sabine L. Flitsch: 0000-0003-3974-646X

\section{Author Contributions}

C.J.G. performed the IM-MS ${ }^{2}$ experiments and analysis. B.S., I.C., and A.M.R. designed and performed the IR-MS ${ }^{2}$ experiments. Computational calculations for the IM-MS ${ }^{2}$ and IR-MS ${ }^{2}$ data were performed by B.S., L.M., A.R.A, and I.C. Synthesis of standards were performed by A.P.G, S.M., and M.S.M. Plant samples were prepared by M.P., R.S.-P., and N.B. All authors contributed to the writing of the manuscript.

Notes

The authors declare no competing financial interest.

\section{ACKNOWLEDGMENTS}

We gratefully acknowledge the Stichting voor Fundamenteel Onderzoek der Materie (FOM) for the support of the FELIX Laboratory, the Villum Research Center for Plant Plasticity, the Center for Synthetic Biology bioSYNergy supported by the University of Copenhagen Excellence Program for Interdisciplinary Research, and the LABEX iMUST (Grant ANR-10-LABX0064) of Université de Lyon, within the program "Investissements d'Avenir" (Grant ANR-11-IDEX-0007) operated by the French National Research Agency (ANR). Travel costs to The Netherlands were supported by the Dutch-French Van Gogh program. In this work, we were granted access to the HPC resources of the FLMSN,"Féderation Lyonnaise de Modélisation et Sciences Numeriques”, partner of EQUIPEX EQUIP@ MESO; HPC CC-IN2P3 at Villeurbanne; and the HPC Resources from GENCI-CINES (Grant 2014-2015 [Grant 087025]). Funding was received from the European Community's Seventh Framework Programme (FP7/20072013) (Grants 312284, 289217, 259869, 278832, 608381, 115360), the BBSRC (Grants BB/F017472/1, BB/L013762/1, $\mathrm{BB} / \mathrm{M} 027791 / 1$ ), and the Royal Society (Wolfson Merit award to S.L.F.). We are indebted to Assoc. Prof. Carl Erik Olsen and Assoc. Prof. Niels Agerbirk for their assistance with preparative HPLC and LC-MS.

\section{REFERENCES}

(1) Both, P.; Green, A. P.; Gray, C. J.; Šardzík, R.; Voglmeir, J.; Fontana, C.; Austeri, M.; Rejzek, M.; Richardson, D.; Field, R. A.; Widmalm, G.; Flitsch, S. L.; Eyers, C. E. Nat. Chem. 2014, 6, 65-74.

(2) Zhu, M.; Bendiak, B.; Clowers, B.; Hill, H., Jr. Anal. Bioanal. Chem. 2009, 394, 1853-1867.

(3) Harvey, D. J.; Scarff, C. A.; Edgeworth, M.; Crispin, M.; Scanlan, C. N.; Sobott, F.; Allman, S.; Baruah, K.; Pritchard, L.; Scrivens, J. H. Electrophoresis 2013, 34, 2368-2378.

(4) Li, H.; Bendiak, B.; Siems, W. F.; Gang, D. R.; Hill, H. H., Jr. Anal. Chem. 2013, 85, 2760-2769. 
(5) Nagy, G.; Pohl, N. B. J. Am. Soc. Mass Spectrom. 2015, 26, 677685.

(6) Nagy, G.; Pohl, N. L. B. Anal. Chem. 2015, 87, 4566-4571.

(7) Bones, J.; Mittermayr, S.; O’Donoghue, N.; Guttman, A.; Rudd, P. M. Anal. Chem. 2010, 82, 10208-10215.

(8) Lee, S.; Wyttenbach, T.; Bowers, M. T. Int. J. Mass Spectrom. Ion Processes 1997, 167-168, 605-614.

(9) Williams, J. P.; Grabenauer, M.; Holland, R. J.; Carpenter, C. J.; Wormald, M. R.; Giles, K.; Harvey, D. J.; Bateman, R. H.; Scrivens, J. H.; Bowers, M. T. Int. J. Mass Spectrom. 2010, 298, 119-127.

(10) Fenn, L. S.; McLean, J. A. Phys. Chem. Chem. Phys. 2011, 13, 2196-2205.

(11) Harvey, D. J.; Scarff, C. A.; Crispin, M.; Scanlan, C. N.; Bonomelli, C.; Scrivens, J. H. J. Am. Soc. Mass Spectrom. 2012, 23, 1955-1966.

(12) Li, H. L.; Bendiak, B.; Siems, W. F.; Gang, D. R.; Hill, H. H. Rapid Commun. Mass Spectrom. 2013, 27, 2699-2709.

(13) Hoffmann, W.; Hofmann, J.; Pagel, K. J. Am. Soc. Mass Spectrom. 2014, 25, 471-479.

(14) Gaye, M. M.; Kurulugama, R.; Clemmer, D. E. Analyst 2015, 140, 6922-6932.

(15) Hofmann, J.; Hahm, H. S.; Seeberger, P. H.; Pagel, K. Nature 2015, 526, 241-244.

(16) Lanucara, F.; Holman, S. W.; Gray, C. J.; Eyers, C. E. Nat. Chem. 2014, 6, 281-294.

(17) Pičmanová, M.; Neilson, E. H.; Motawia, M. S.; Olsen, C. E.; Agerbirk, N.; Gray, C. J.; Flitsch, S.; Meier, S.; Silvestro, D.; Jørgensen, K.; Sánchez Pérez, R.; Møller, B. L.; Bjarnholt, N. Biochem. J. 2015, 469, 375-389.

(18) Campuzano, I.; Bush, M. F.; Robinson, C. V.; Beaumont, C.; Richardson, K.; Kim, H.; Kim, H. I. Anal. Chem. 2012, 84, 1026-1033.

(19) Konda, C.; Bendiak, B.; Xia, Y. J. Am. Soc. Mass Spectrom. 2012, 23, 347-358.

(20) Domon, B.; Costello, C. E. Glycoconjugate J. 1988, 5, 397-409.

(21) Cancilla, M. T.; Wang, A. W.; Voss, L. R.; Lebrilla, C. B. Anal. Chem. 1999, 71, 3206-3218.

(22) Cancilla, M. T.; Penn, S. G.; Carroll, J. A.; Lebrilla, C. B. J. Am. Chem. Soc. 1996, 118, 6736-6745.

(23) Zaia, J. Mass Spectrom. Rev. 2004, 23, 161-227.

(24) Rijs, A. M.; Oomens, J. In Gas-Phase IR Spectroscopy and Structure of Biological Molecules; Rijs, M. A., Oomens, J., Eds.; Springer International Publishing: Cham, Switzerland, 2015; pp 1-42.

(25) Stefan, S. E.; Eyler, J. R. Int. J. Mass Spectrom. 2010, 297, 96101.

(26) Barnes, L.; Schindler, B.; Allouche, A.-R.; Simon, D.; Chambert, S.; Oomens, J.; Compagnon, I. Phys. Chem. Chem. Phys. 2015, 17, 25705-25713.

(27) Brown, D. J.; Stefan, S. E.; Berden, G.; Steill, J. D.; Oomens, J.; Eyler, J. R.; Bendiak, B. Carbohydr. Res. 2011, 346, 2469-2481.

(28) Schindler, B.; Joshi, J.; Allouche, A. R.; Simon, D.; Chambert, S.; Brites, V.; Gaigeot, M. P.; Compagnon, I. Phys. Chem. Chem. Phys. 2014, 16, 22131-22138.

(29) Polfer, N. C.; Valle, J. J.; Moore, D. T.; Oomens, J.; Eyler, J. R.; Bendiak, B. Anal. Chem. 2006, 78, 670-679.

(30) Hernandez, O.; Isenberg, S.; Steinmetz, V.; Glish, G. L.; Maitre, P. J. Phys. Chem. A 2015, 119, 6057-6064.

(31) Tan, Y.; Polfer, N. J. Am. Soc. Mass Spectrom. 2015, 26, 359368. 